cannot fuse their mitochondria. These fusion-deficient cells display slow growth, decreased mitochondrial membrane potential, and impaired respiratory capacity. These defects are reversed upon restoration of fusion capability. The importance of mitochondrial fusion is further underscored by the fact that two neurodegenerative diseases, CharcotMarie-Tooth disease type 2A (CMT) and autosomal dominant optic atrophy (DOA), are caused by mutations in Mfn2 and OPA1, respectively.To further explore the physiological requirements for mitochondrial fusion, we have created conditional knockout mice for both $\mathrm{Mfn} 1$ and $\mathrm{Mfn} 2$. Interestingly, Mfn2 is required for proper cerebellar development. When cerebellar primordia is targeted embryonically, pups display severe problems in balance and motility, resulting in feeding problems and death by about two weeks of age. When Purkinje cells, the only efferent processes of the cerebellum, are targeted upon maturity, they actually degenerate, causing the mice to develop tremors and wobble while walking. Thus, mitochondrial fusion is required in the central nervous system along with the peripheral motor neurons and optic nerve affected in CMT and DOA.Disruption of both Mfn1 and Mfn2 in skeletal muscle results in severely runted mice, which die at around two months of age. Muscle mass is greatly reduced in these mice, and cross-sectional analysis reveals significantly decreased muscle fiber widths. A modified Gomori-trichrome stain results in predominantly red fibers. In addition, a succinate-dehydrogenase (SDH) enzymatic assay reveals greatly increased activity in all fibers. The combination of red fibers and increased SDH activity is a hallmark of many mitochondrial diseases, suggesting a common link between deficiencies in mitochondrial fusion and traditional mitochondrial diseases.

doi:10.1016/j.mito.2006.08.012

Peroxisomal proliferator activated receptor agonists as potential therapy for mitochondrial respiratory chain complex (RCC) defects

Joseph C. Huey, Bambi D. Anderson, Maureen M. Black, Dietrich Matern, Sihoun Hahn *

Department of Laboratory Medicine and Pathology, Pediatric and Adolescent Medicine, Medical Genetics, Mayo Clinic College of Medicine, Rochester, MN, USA

Peroxisome proliferator activated receptors (PPARs) are a family of ligand-activated transcription factors involved in lipid and glucose metabolism, immune regulation, and cell differentiation. PPAR $\alpha$ is preferentially expressed in tissues with high fatty acid utilization like heart and muscle, while PPAR $\gamma$ is found at high levels in adipocytes and other cell types involved in lipid synthesis and storage. Recent data highlighted the potential of fibrates, PPAR $\alpha$ agonists, in the correction of inborn errors of fatty acid oxidation. We investigated the effects of Bezafibrate, a common hypolipidemic drug acting as a PPAR $\alpha$ agonist, Pioglitazone and Rosiglitazone, PPAR $\gamma$ agonists used for non-insulin-dependent diabetes on mitochondrial RCC capacities in complex I and IV deficient cell lines. Skin fibroblasts from patients with established defects of complex I, IV and normal controls were incubated for $72 \mathrm{~h}$ with $500 \mu \mathrm{M}-1 \mathrm{mM}$ Bezafibrate, 100-400 nM Rosiglitazone and Pioglitazone, respectively, or the equivalent amount of DMSO (vehicle). Fibroblasts were harvested and assayed for complex I, II + III, IV, and citrate synthase. The complex IV enzyme activities in two definite COX deficient cell lines remained unchanged after the treatments even at very high concentrations. The changes in three definite complex I deficiencies were also minimum while complex I enzyme activities in two partial deficient cell lines increased up to nearly $50 \%$ after the PPAR $\gamma$ treatment. The citrate synthase tended to increase after the treatment with both PPAR $\alpha$ and PPAR $\gamma$ suggesting mitochondrial proliferation. Our data provides the foundation for possible therapeutic intervention for the patients with mitochondrial disorders, especially for those with partially deficient RCC enzymes. Given the skin fibroblasts are not mitochondria-rich tissue, the response in muscle tissues to PPAR agonists could be more favorable. Further study on a variety of proven deficient cell lines will be necessary to prove the effectiveness of the PPAR agonists in mitochondrial disorders.

doi:10.1016/j.mito.2006.08.013

\section{Biochemical consequences of a pathogenic A3243G mtDNA} mutation

J.A. Mayr a , F. Zimmermann, D. Meierhofer, N. Schmeller ${ }^{\mathrm{b}}$, W. Sperl ${ }^{\mathrm{a}}$, B. Kofler ${ }^{\mathrm{a},{ }^{*}}$

a Department of Pediatrics, Paracelsus Private Medical University Salzburg, Austria; ${ }^{\mathrm{b}}$ Department of Urology, Paracelsus Private Medical University Salzburg, Austria

To elucidate the relationship between tumorgenesis and alterations of the respiratory chain in renal tumors, we screened renal tumors (malignat renal cell carcinomas and benign oncocytomas) and the corresponding normal tissue for somatic mitochondrial DNA (mtDNA) variations by denaturing HPLC (DHPLC) analysis and measured enzyme activities of the oxidative phosphorylation (OXPHOS) and the Krebs-cycle (Br. J. Cancer 2006. 94, 268-74). Sequencing of somatic mutations revealed in one renal cell carcinoma and one renal oncocytoma an A to $G$ transition at position 3243, which is known as a frequent cause of MELAS (Mitochondrial Encephalomyopathy with Lactic Acidosis and Stroke-like episodes). RFLP analysis revealed $>95 \%$ mutational load in the oncocytoma tissue but also a preexisting mutation in the normal kidney tissue of $20 \%$. In the case of the renal cell carcinoma ( $89 \%$ heteroplasmy) the corresponding kidney tissue was unaffected. Upregulation of the nuclear encoded enzyme activities of citrate synthase, isocitrate dehydrogenase, and oligomycine-sensitive ATPase and downregulation of 
mixed nuclear and mitochondrial encoded OXPHOS complexes I, III, and IV was detected. Interestingly, the complex I activity was more severely impaired than the complex IV activity. The biochemical consequences of the A3243G mutation in the two tumor tissues are in agreement with the decreased activity of the mitochondrial and nuclear encoded complex I and IV reported in muscle biopsy and fibroblasts of patients with MELAS syndrome. The A3243G mutation might be sporadically associated with other tumor types since it was also detected in a colon cancer sample (Mitochondrion 2003, 3, 119-124). Furthermore, the presence of the pathogenic A3243G mutation in the oncocytoma tissue can explain the compensatory hyperproliferation of mitochondria, which is characteristic for oxyphilic tumors.

doi:10.1016/j.mito.2006.08.014

Multiplex primer extension analysis for rapid detection of major European mitochondrial haplogroups

Martina Wiesbauer ${ }^{\mathrm{b}}$, David Meierhofer ${ }^{\mathrm{b}}$, Johannes A. Mayr ${ }^{\mathrm{b}}$, Wolfgang Sper $^{\mathrm{a}, \mathrm{b}}$, Bernhard Paulweber ${ }^{\mathrm{a}, \mathrm{b}}$, Barbara Kofler b,*

${ }^{a}$ Department of Internal Medicine I, Paracelsus Private Medical University Salzburg, Muellner Hauptstrasse 48, A-5020 Salzburg, Austria; ${ }^{\mathrm{b}}$ Department of Pediatrics, Paracelsus Private Medical University Salzburg, Muellner Hauptstrasse 48, A-5020 Salzburg, Austria

Alterations of mitochondrial DNA (mtDNA) have been instrumental in studies of human evolution, in population genetics, and in molecular medicine to link mtDNA variations to a variety of human diseases of complex etiology such as asthenozoospermia, multiple sclerosis, optical stroke, and Parkinson. For each of these applications, rapid and cost effective assays for mtDNA haplogrouping are invaluable. Here we describe a hierarchical system for mtDNA haplogrouping that combines multiplex PCR amplifications, multiplex single-base primer extensions, and capillary-based electrophoretic separation for analysing ten haplogroup-diagnostic mitochondrial single nucleotide polymorphisms (mtSNPs). Specific primers were designed to amplify the mtDNA regions containing the polymorphic sites that characterise each of the major European haplogroups $(\mathrm{H}, \mathrm{I}, \mathrm{J}, \mathrm{K}, \mathrm{T}, \mathrm{U}, \mathrm{V}, \mathrm{W}, \mathrm{X})$ in two multiplex PCRs, A and B. Assay A was designed to determine the most common European haplogroups, H, J, K, T, U, and included primers to amplify five PCR fragments encompassing six informative mtSNPs. Assay B, containing four mtSNPs, was only used for the remaining haplogroups $\mathrm{V}, \mathrm{W}, \mathrm{I}, \mathrm{X}$, not identified by assay A. To enable electrophoretic resolution of the different primer extension products, poly-adenosine tails 5-25 nucleotides in length were included at the $5^{\prime}$ end of the SNP primers. Using this rapid and cost-effective mtDNA genotyping method, we were able to show that within a large, randomly selected cohort of healthy Austrians $(n=1172)$, mtDNAs could be assigned to all nine major European haplogroups. Forty-four percent belonged to haplogroup $\mathrm{H}$, the most frequent haplogroup in European Caucasian populations. The other major haplogroups identified were U (15.4\%), $\mathrm{J}(11.8 \%), \mathrm{T}(8.2 \%)$, and $\mathrm{K}(5.1 \%)$. The frequencies of haplogroups in Austria are within the range observed for other European countries. Our method may be suitable for mitochondrial genotyping of samples from large-scale epidemiology studies and for identifying markers of genetic susceptibility.

doi:10.1016/j.mito.2006.08.015

NMR investigation of metabolism in cultured human fibroblasts: Effects of PDC deficiency and DCA administration P.W. Stacpoole, N.E. Simpson ", Z. Han, K.M. Berendzen, C.A. Sweeney, J.A. Oca-Cossio, I. Constantinidis University of Florida, College of Medicine, USA

The pyruvate dehydrogenase complex (PDC) is integral to mitochondrial metabolism and energetics. Congenital PDC deficiency leads to lactic acidosis, neurological degeneration and early death. Dichloroacetate (DCA), an investigational compound for such defects, activates the PDC by inhibiting the reversible phosphorylation of the Ela subunit and decreases its turnover. Primary human fibroblast cultures from 5 controls and 6 patients with mutations in the PDC-E1 component were grown in media $\pm 5 \mathrm{mM}$ DCA, exposed to media containing $15 \mathrm{mM} \mathrm{13C-labeled}$ glucose, with the aqueous phase of the cell extracts studied by nuclear magnetic resonance (NMR) spectroscopy. Computer modeling of NMR-derived 13C-glutamate isotopomeric patterns estimated relative carbon flow through tricarboxylic acid cycle-associated pathways and characterized effects of PDC deficiency on glucose metabolism and energetics. With the exception of one patient cell-line expressing an unusual splicing mutation, PDC-deficient cells had significantly higher glucose consumption, lactate production, and label-derived acetyl-CoA, indicative of increased glycolysis vs. controls. In all cells, DCA caused a major shift (40\% decrease) from anaplerotic pathways (e.g., pyruvate carboxylase) toward flux through PDC. Ignoring the mis-spliced patient, DCA decreased average glycolysis $(29 \%)$ in patient cells, but had no effect in control cells, and did not change lactate production or the nucleoside triphosphate to diphosphate ratio (NTP/NDP) in either cell group. Maintenance of NTP despite reduced glycolysis indicates that DCA improved metabolic efficiency by increasing glucose oxidation. This study demonstrates that NMR spectroscopy provides novel insight into the biochemical consequences of PDC deficiency and the mechanism of putative therapeutic agents thereon.

doi:10.1016/j.mito.2006.08.016 